\section{P2-254 EXPOSURE TO TRAFFIC AND CANCER HOSPITALISATION IN São PAULO}

doi:10.1136/jech.2011.142976j.87

A Ribeiro, ${ }^{*}$ A Nardocci. University of São Paulo, São Paulo, Brazil

Introduction Previous studies in developed countries have documented associations between air pollution and risk of some neoplasms. We explored the association of traffic-related air pollution with hospitalisations for cancer groups.

Methods Our analysis included all individuals admitted to public or private hospitals in São Paulo from 2004 to 2006 with a main diagnosis of primary invasive cancer. Only the first individual admission was considered, from which age, sex, diagnosis and home address were extracted. We calculated total, gasoline and diesel vehicles traffic density, from traffic counts data, for 4964 geographical units with a population of 20 or more inhabitants, formed by a grid of 500 by $500 \mathrm{~m}$. We used logistic regression models adjusted by the Human Development Index of the area for groups of cancer.

Results There was an increased risk of hospitalisation for respiratory neoplasms in adults and for haematologic neoplasms in children and adolescents associated with living in areas with higher total traffic density and traffic density for vehicles powered by gasoline and diesel, with a clear dose-response gradient. The Rate ratios of these neoplasms for the highest category of exposure to total density traffic were, respectively, 3.31 (95\% CI 2.26 to 4.87 ) and 2.35 (95\% CI 1.59 to 3.49 ).

Conclusion Our study suggests an association between traffic air pollution and hospital admissions for respiratory and haematologic cancers. The adjustment for potential confounding variables, the use of more sophisticated exposure assessment models and of incidence data are needed to more directly investigate the cause and effect relationship.

\section{P2-255 TRENDS IN MORTALITY DUE TO POTENTIALLY HPV- RELATED HEAD AND NECK CANCERS IN BRAZIL, 1980-2007}

doi:10.1136/jech.2011.142976j.88

A Manso, I Oliveira, F Yazawa, M A Veras, K Ribeiro. * Faculdade de Ciências Médicas da Santa Casa de São Paulo, São Paulo, Brazil

Introduction Alcohol and tobacco are the most important risk factors for head and neck cancers (HNC). However, in some countries, it has been observed an increase in incidence rates, particularly among young people and among non-drinkers and non-smokers. These trends have increased the debate about the role of human papillomavirus (HPV) and several studies suggest that this infection may play a causative role in oropharyngeal tumours. This study aims to assess the magnitude of cancers potentially related to HPV in Brazil. Methods Time series study including deaths due to HNC registered in Brazil, from 1980 to 2007. Age-adjusted mortality rates are described for 100000 people, according to tumour site (HPV-related or non-HPV related) and sex. Annual percentage change (APC) was calculated through Joinpoint modeling method, using the calendar year as regressor variable.

Results We observed a statistically significant increasing trends in mortality rates due to HPV-related HNC among males in the periods of 1980-1994 (APC $=2.4,95 \%$ CI 1.0 to 3.8) and 1997-2005 (APC $=4.0,95 \%$ CI 0.8 to 7.4), followed by a decrease in 2005-2007 (APC $=-27.2,95 \% \mathrm{CI}-44.6$ to -4.4 ), while for females a significant increase in rates was observed between 1980 and 1998 (APC=7.6, $95 \%$ CI 0.3 to 15.4 ), followed by a decreasing trend in the period 1998-2007 (APC $=-2.7,95 \% \mathrm{CI}-4.2$ to -1.3 ). Regarding non-HPV related HNC, significant changes were only observed for females (1980-2007, APC $=-2.1,95 \%$ CI -2.8 to -1.4$)$.

Conclusion Important decreases in HPV-related HNC mortality were recently observed in Brazil, particularly among females. These results can suggest that these trends reflects better prognosis related to HPV-positive HNC.

\section{P2-256 DESCRIPTIVE DATA ON WORK-RELATED CANCER IN BRAZIL: AN ANALYSIS WITH COMPENSATION BENEFITS}

doi:10.1136/jech.2011.142976j.89

${ }^{1} \mathrm{~F}$ Ribeiro, ${ }^{2} \mathrm{~V}$ Santana, ${ }^{3} \mathrm{~W}$ Souza. ${ }^{1}$ Rio de Janeiro State University and National Cancer Institute, Rio de Janeiro, Rio de Janeiro, Brazil, '2Institute of Collective Health, Federal University of Bahia, Salvador, Bahia, Brazil; ${ }^{3}$ University of Brasilia, Brasilia, Distrito Federal, Brazil

In Brazil, data on work-related diseases and injuries are commonly recorded by the Brazilian Social Security Institute, INSS, which covered $32 \%$ of the economically active population, approximately 32 million workers, in 2009. Industries known to be associated with potential exposure of workers to carcinogenic substances are spread throughout the country and account for a substantial proportion of the labour force. There are no published work-related cancer statistics. This study describes the national distribution of occupational cancer cases leading to compensation benefit in Brazil, from 2000 and 2007

Methods The study population comprised of all insured workers eligible for work-related compensation benefits, recorded electronically in the INSS. Cases were workers who received a compensation benefit classified as work-related and ICD-10 (C30-C96). Descriptive variables were sex, age, income, state, industry trades and year of the benefit

Results The number of registered benefits for cancer cases increased from 33488 to 43464; from these 724 (0.4\%) work-related cancer cases were found. Most were $<35$ years of age and from the first income quartile. Cases were more likely to come from the construction industry, and leukaemia and other haematopoietic cancers predominated. The number of reported work-related cases declined from 100 in 2000 to 34 in 2006, when they started to increase reaching 266 in 2007.

Conclusions In Brazil, the number of reported cases of work-related cancers was small and decreasing during the last decade. After the implementation of a distinct work-relatedness protocol in 2007 it changed, increasing over $100 \%$, suggesting large under reporting. Epidemiological estimates with these data are presently undergoing.

\section{P2-257 SOCIAL DETERMINANTS AND HIGH RISK BEHAVIOURS IN DRUG USERS UNDER METHADONE TREATMENT IN GOLESTAN PROVINCE, NORTH IRAN}

doi:10.1136/jech.2011.142976j.90

${ }^{1} \mathrm{M}$ Gholipour, ${ }^{* 1,2} \mathrm{~A}$ Keshtkar, ${ }^{3} \mathrm{R}$ Majdzadeh, ${ }^{4} \mathrm{~A}$ Badakhshan, ${ }^{3} \mathrm{~S}$ nejat, ${ }^{1,5} \mathrm{M}$ Oorbani, ${ }^{1} \mathrm{M}$ Vakili, ${ }^{6} \mathrm{H}$ Salari. ${ }^{1}$ Community Medicine Department, School of Medicine, Golestan University of Medical Sciences, Gorgan, Golestan, Iran; ${ }^{2}$ Endocrinology Metabolism Research Centre, Tehran University of Medical Sciences, Tehran, Iran; ${ }^{3}$ Epidemiology and Biostatistics Department, School of Public Health, Tehran University of Medical Sciences, Tehran, Iran; ${ }^{4}$ Deputy of treatment, Golestan University of Medical Sciences, Gorgan, Iran; ${ }^{5}$ Iran university of Medical Sciences, Tehran, Iran; ${ }^{6}$ Psychiatric Department, School of Medicine, Golestan University of Medical Sciences, Gorgan, Golestan, Iran

Introduction Drug users' health is a product of drug-effects and risk behaviours. Social determinants can shape some health behaviours. There has been little research on social determinants and risk behaviours related to drug use in Iran. We aimed to estimate this in patients undergoing methadone treatment in north Iran. 
Methods 400 addicts were recruited from methadone clinics in 2009-2010. A self-designed questionnaire with excellent reliability was used to determine those with and without high risk behaviours. ORs with $95 \%$ CIs were estimated by logistic regression. Ethics approval was obtained from Tehran University.

Results There was significant difference between the two study groups (with and without high risk behaviours) in economic status, drug type, administration route, age, and drug abuse onset age. Education level was significantly lower in women. One quarter experienced homeless, $62 \%$ had no support from any insurance or supportive organisations, only $26 \%$ had constant employment and over $50 \%$ had prison history. $12.5 \%$ were injection users and $14 \%$ shared syringes. One quarter reported high risk sexual behaviour and $69.4 \%$ had not used condoms in last their last sex encounter (significantly lower in women). A decrease of one year in age was associated with decreased drug use onset age and increased sexual risk behaviours by $6 \%(\mathrm{AOR}=0.94,95 \% \mathrm{CI} 0.91$ to 0.98$)$ and $10 \%$ (AOR $=0.91,95 \%$ CI 0.85 to 0.97 ) respectively. Poor economic status reduced risk sexual behaviour ( $\mathrm{AOR}=0.35,95 \%$ CI 0.13 to 0.96 ). Prison history increased injection behaviour more than twice (AOR $=2.89,95 \%$ CI 1.4 to 5.95 )

Conclusions These findings illustrate that interventions are needed in young heroin users even in those with a good economic state.

\section{P2-258 EVALUATION OF THE EFFECTIVENESS OF THE PHARMACOTHERAPY FOLLOW-UP ON THE TREATMENT OF HYPERTENSIVE PATIENTS: A COHORT STUDY}

\section{doi:10.1136/jech.2011.142976j.91}

${ }^{1} \mathrm{~F}$ Rodrigues, ${ }^{2,3} \mathrm{M}$ Castro, ${ }^{1,2} \mathrm{~S}$ Fuchs, ${ }^{1,2}$ F Fuchs, ${ }^{1,2}$ L Moreira. ${ }^{* 1}$ Postgraduate Studies Program in Health Sciences: Cardiology and Cardiovascular Sciences; UFRGS, Porto Alegre, Rio Grande do Sul, Brazil; ${ }^{2}$ Division of Cardiology, Hospital de Clínicas de Porto Alegre, Porto Alegre, Rio Grande do Sul, Brazil; ${ }^{3}$ Pharmacy School, UFRGS, Porto Alegre, Rio Grande do Sul, Brazil

Introduction The control rate of hypertension in the population is fair. Pharmaceutical Care is a recent approach, seeking to magnify therapeutic results.

Objective To evaluate the effectiveness of the pharmacotherapy follow-up conducted by the Pharmacist on hypertension management.

Methods The study is a historical cohort with a dynamic population of patients referred to an outpatient hypertension clinic. Patients were followed for 12 months. Those difficult-to-control referred by the physician to pharmacotherapy follow-up were compared with patients under conventional treatment. Endpoints included blood pressure (BP) variation and the rate of control $(<140 / 90 \mathrm{~mm} \mathrm{Hg})$. General Linear Model, modified Poisson Regression, and segmented regression were used in the data analysis.

Results Of 993 patients, 150 were referred for pharmacotherapy follow-up. Patients in the pharmacotherapy follow-up were older, with lower education level, longer diagnoses of hypertension and significantly higher levels of BP. The deltas of SBP were $7.4 \pm 1.9$ vs $10.3 \pm 0.8 \mathrm{~mm} \mathrm{Hg} \quad(p=0.16)$ and diastolic $6.7 \pm 1.0 \mathrm{~mm} \mathrm{Hg}$ vs $5.9 \pm 0.4(p=0.45)$ for pharmacotherapy follow-up and conventional treatment groups, respectively, adjusted for initial pressure. The control rate was $45.3 \%$, being $28.1 \%$ in the exposed and $48.6 \%$ in the unexposed $(p<0.001)$. Comparing the BP of the same patients before and after exposure to pharmacotherapy follow-up showed a change in the trend of SBP and DBP $(p<0.001)$.

Conclusion Hypertensive patient difficult-to-control under pharmacotherapy follow-up showed a reduction in BP similar to patients who received only conventional treatment. The study suggests that pharmacotherapy follow-up is effective in the management of selected hypertensive patients.

\section{P2-259 CANCER RISK IN CHILDREN WITH BIRTH DEFECTS: A LONGITUDINAL, POPULATION-BASED ASSESSMENT AMONG 2.7 MILLION BIRTHS}

doi:10.1136/jech.2011.142976j.92

${ }^{1} \mathrm{P}$ A Romitti, ${ }^{2} \mathrm{~T}$ Flood, ${ }^{3} \mathrm{M}$ L Feldkamp, ${ }^{3} \mathrm{~S}$ Krikov, ${ }^{1} \mathrm{~S}$ Puzhankara, ${ }^{1} \mathrm{R}$ Goedken, ${ }^{3} \mathrm{M}$ Fluchel, ${ }^{4} \mathrm{~J}$ Little, ${ }^{3} \mathrm{~L} \mathrm{D}$ Botto. ${ }^{1}$ The University of lowa, lowa City, lowa, USA; ${ }^{2}$ Arizona Department of Public Health, Phoenix, Arizona, USA; ${ }^{3}$ University of Utah, Salt Lake City, Utah, USA; ${ }^{4}$ University of Ottawa, Ottawa, Ontario, Canada

Introduction The published literature, to date, is largely inconclusive regarding cancer risk among children with birth defects. To improve knowledge of such risk, we studied population-based (statewide) birth cohorts from Arizona, Iowa, and Utah selected from among 2.7 million births delivered from 1983 to 2006

Methods Birth defect and cancer diagnoses were identified from linked population-based surveillance systems. A population-based cohort of over 43000 children with major birth defects (including trisomies 13, 18, and 21) was compared to a cohort of nearly 148000 births without birth defects, randomly sampled from the same underlying birth population and frequency-matched to the birth defects cohort by birth year. Kaplan-Meier time-to-event analysis, accounting for censoring by death, was used to estimate cancer risk up to age 15 years.

Results Compared to the reference cohort, children with birth defects had a statistically significant increase in cancer risk ( $R R$, 2.73). Risk was highest among children with Down syndrome (RR, 13.2), and was driven largely by leukaemias. Cancer risk was moderately increased among children with a birth defect but without chromosomal anomalies (RR, 1.82). In this group, cancer risk was driven largely by brain tumours and embryonal tumours, and occurred mainly in children with brain defects, cleft palate, rectal defects, and some heart defects.

Conclusion These population-based findings support and extend previous findings that suggest increased cancer risk in children with birth defects, including non-chromosomal defects, and suggest selected defect groups in which further research could help identify a common genetic susceptibility to cancer and birth defects.

\section{P2-260 POPULATION-BASED PREVALENCE OF DUCHENNE/BECKER MUSCULAR DYSTROPHY (DBMD) IN THE USA}

doi:10.1136/jech.2011.142976j.93

${ }^{1} \mathrm{P}$ Romitti, ${ }^{1} \mathrm{~S}$ Puzhankara, ${ }^{1} \mathrm{G}$ Zamba, ${ }^{1} \mathrm{~S}$ Nabukera, ${ }^{5} \mathrm{~K}$ James, ${ }^{3} \mathrm{~J}$ Andrews, ${ }^{2} \mathrm{D}$ Fox, ${ }^{3} \mathrm{C}$ Cunniff, ${ }^{4} \mathrm{E}$ Ciafaloni, ${ }^{2} \mathrm{C}$ Druschel, ${ }^{1} \mathrm{~K}$ Mathews, ${ }^{5} \mathrm{D}$ Matthews, ${ }^{6} \mathrm{~L}$ Miller, ${ }^{4} \mathrm{~S}$ Pandya, ${ }^{7} \mathrm{~S} \mathrm{Au},{ }^{7} \mathrm{~S}$ Scollon, ${ }^{8} \mathrm{M}$ Adams, ${ }^{8} \mathrm{~N}$ Street, ${ }^{8}$ the Muscular Dystrophy Surveillance, Tracking, Research Network (MD STARnet). 'The University of lowa, lowa City, lowa, USA; ${ }^{2}$ New York State Department of Health, Troy, New York, USA; ${ }^{3}$ University of Arizona, Tucson, Arizona, USA; ${ }^{4}$ University of Rochester, Rochester, New York, USA; ${ }^{5}$ University of Colorado, Denver, Colorado, USA; ${ }^{6}$ Colorado Department of Public Health and Environment, Denver, Colorado, USA; ${ }^{7}$ Hawaii Department of Health, Honolulu, Hawaii, USA; ${ }^{8}$ Centers for Disease Control and Prevention, Atlanta, Georgia, USA

Introduction DBMD has an estimated prevalence of 1/3500 male births. Worldwide, this estimate varies, likely due to differences in diagnostic criteria, ascertainment, and survival. To date, no U.S. population-based DBMD prevalence data by race/ethnicity have been published.

Methods In 2002, the Centers for Disease Control and Prevention established the MD STARnet to conduct population-based DBMD surveillance in four U.S. sites. Each site conducts active surveillance to identify males with DBMD born since January 1982 . Using these data, we calculated DBMD prevalence by race/ethnic subgroups and birth intervals (1986-1990; 1991-1995; 1996-2000). Prevalence was calculated as: [number of DBMD males age 5-9 years/number of male residents, age 5-9 years]. With the average age at DBMD 